\title{
Effects of androgen deprivation on brain function in prostate cancer patients - a prospective observational cohort analysis
}

\author{
Herta H Chao ${ }^{1,2,9^{*}}$, Edward Uchio ${ }^{3}$, Sheng Zhang ${ }^{4}$, Sien Hu${ }^{4}$, Sarah R Bednarski ${ }^{4}, X_{i}$ Luo $^{5}$, Michal Rose ${ }^{1,2}$,
} John Concato ${ }^{1,2,6}$ and Chiang-shan R Li $i^{4,7,8}$

\begin{abstract}
Background: Despite a lack of consensus regarding effectiveness, androgen deprivation therapy (ADT) is a common treatment for non-metastatic, low-risk prostate cancer. To examine a particular clinical concern regarding the possible impact of ADT on cognition, the current study combined neuropsychological testing with functional magnetic resonance imaging (fMRI) to assess both brain activation during cognitive performance as well as the integrity of brain connectivity.

Methods: In a prospective observational cohort analysis of men with non-metastatic prostate cancer at a Veterans Affairs medical center, patients receiving ADT were compared with patients not receiving ADT at baseline and at 6 months. Assessments included fMRI, the N-back task (for working memory), the stop-signal task (for cognitive control), and a quality of life questionnaire.

Results: Among 36 patients enrolled (18 in each group), 30 completed study evaluations (15 in each group); 5 withdrew participation and 1 died. Results for the N-back task, stop-signal task, and quality of life were similar at 6 months vs. baseline in each group. In contrast, statistically significant associations were found between ADT use (vs. non use) and decreased medial prefrontal cortical activation during cognitive control, as well as decreased connectivity between the medial prefrontal cortex and other regions involved with cognitive control.

Conclusions: Although ADT for 6 months did not affect selected tests of cognitive function, brain activations during cognitive control and functional brain connectivity were impaired on fMRI. The long-term clinical implications of these changes are not known and warrant future study.
\end{abstract}

Keywords: Androgen deprivation, Prostate cancer, Brain function, Cognitive function

\section{Background}

Prostate cancer is the most common non-skin cancer in American men, and almost half of patients with this disease receive androgen deprivation therapy (ADT) over the course of their disease [1,2]. Evidence suggests that ADT can cause fatigue, decreased sexual function, gynecomastia, osteoporosis, and metabolic changes [3], but the adverse effects of ADT on cognition remain unclear. Observational and randomized studies have

\footnotetext{
* Correspondence: herta.chao@yale.edu

'Department of Internal Medicine \& Yale Comprehensive Cancer Center, Yale University School of Medicine, New Haven, CT 06519, USA

${ }^{2}$ Medical Service, VA Connecticut Health Care System, West Haven, CT 06516, USA

Full list of author information is available at the end of the article
}

examined a possible association between androgens and cognitive function, including a potentially protective effect of testosterone against age-related cognitive decline [3-8]. A review [9] of prior studies using traditional neuropsychological testing in patients with prostate cancer treated with ADT showed equivocal findings; results were interpreted as showing either no effect [10], impaired function [11-13], or a mixed effect of ADT (with patients improving on some tests but doing worse on others) [14].

Functional magnetic resonance imaging (fMRI) provides a non-invasive method to assess brain activations during cognitive performance as well as the integrity of regional functional connectivities at rest. Clinicians have

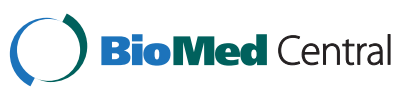


used fMRI to understand whether specific brain processes are impaired in patients with a medical (e.g., congenital heart disease [15]) or neurological condition (e.g., cerebral palsy [16]). Studies using fMRI have demonstrated etiology-specific changes in regional brain activations in patients with neurological or psychiatric conditions, even when they did not score differently from control individuals in neurocognitive tasks [17-19]. Thus, fMRI provides a more sensitive measure of brain functioning than neurocognitive testing alone.

Investigators have used fMRI and other imaging methods to evaluate the effects of chemotherapy and hormonal therapy on brain function in women with breast cancer [20]. For example, altered cortical and subcortical metabolism was found in breast cancer survivors 510 years after receiving adjuvant chemotherapy [21], and a patient who was diagnosed with breast cancer and underwent chemotherapy showed more white matter hyperintensities and altered spatial extents of brain activation than her non-affected, monozygotic twin, despite only small differences in working memory performance [22]. In addition, women with breast cancer taking tamoxifen showed widespread cortical hypometabolism on PET and MRI, when compared with scans in women not taking tamoxifen [23]. A longitudinal study in premenopausal women with early-stage breast cancer demonstrated changes in cognitive functioning and cerebral white matter integrity after chemotherapy [24]. Another recent study demonstrated significantly reduced activation in the left middle dorsolateral prefrontal cortex and premotor cortex in women with breast cancer compared with healthy controls irrespective of treatment history [25].

Our objective was to evaluate the impact of ADT on cognition using fMRI to study brain activations during a cognitive task and at rest, the stop-signal-task to study cognitive control, and the N-back test to study working memory. As a secondary outcome, we also evaluated quality of life.

\section{Methods}

\section{Participants and baseline clinical assessments}

First, patients with non-metastatic prostate cancer at the VA Connecticut Healthcare System from 01/2009 through 12/2010 were identified. All men who were prescribed ADT-either as adjuvant treatment or because of biochemical recurrence-were approached for participation. As per clinical practice, ADT consisted of medical castration with Goserelin $10.8 \mathrm{mg}$ subcutaneously every 90 days for 6 months, after a lead-in period with Bicalutamide $50 \mathrm{mg}$ daily. Patients with non-metastatic prostate cancer who had never been treated with ADT were evaluated as potential controls, with matching based on age and level of education. Exclusion criteria were: active second malignancy; Eastern Cooperative Oncology Group Performance Status $>1$; any significant cardiovascular conditions (e.g., unstable angina, pacemaker); hepatic, renal, or neurological disease; any investigational agents; or a score of less than 27 out of 30 on the mini-mental state examination (MMSE) [26]. Patients who had a history of axis-I psychiatric or substance (excluding nicotine) use disorders [27] were also excluded. All participants underwent a health questionnaire interview to ensure eligibility for fMRI. Participants who had had a prostatectomy were at least 3 months from their surgery and had fully recovered before study entry. Participants who were to receive radiation to the prostate underwent baseline assessment before starting any treatment and were also evaluated 3 to 4 months after completion of radiation treatment.

Our study was approved by the Yale Human Investigation Committee and the Human Investigation Subcommittee of the Veterans Affairs (VA) Connecticut Health Care System.

\section{Assessments}

The ADT-treated participants were studied prior to initiation of ADT and again after 6 months of ADT, with effective castration documented by measuring testosterone level. Each individual assessment (questionnaire, performance-based, or radiological), as well as the combination of assessments (consisting of cognitive testing during a fMRI scan), are described below.

\section{Quality of life}

As a general assessment of overall status, participants completed standardized Quality-of-Life-Questionnaires (QOL) for prostate cancer patients $\left(\right.$ Fact- $\left.\mathrm{P}^{\odot}\right)$ at baseline and again at 6 months [28].

\section{$\mathrm{N}$-back (working memory) task}

Working memory is a form of short-term memory that allows individuals to retain and manipulate information concurrent with engaging in complex tasks such as comprehension, reasoning, and learning. A behavioral paradigm widely used to study working memory is the N-back task $[29,30]$. In response to a series of letters displayed at a rate of 1 every 2 seconds, a higher accuracy rate and shorter reaction (RT) of correct trials represents better working memory. Our participants performed an N-back working memory task outside the MRI scanner at baseline and also at 6 months.

\section{Stop signal (cognitive control) task}

The stop signal task has been validated as a method to study cognitive control [31,32]. The stop signal task has two different stimuli randomly mixed in presentation and time intervals: a "Go" stimulus that instructs 
participants to respond by pressing a button, and a "Stop" stimulus that instructs participants to withhold their response. Pressing a button prematurely, prior to the appearance of the "Go" stimulus, terminates a trial. Likewise, a trial terminates at button press or when 1 second has elapsed after the appearance of the stop signal. A greater accuracy rate of "Go" and "Stop" trials indicates better cognitive control. Furthermore, in the stop signal task, participants can slow down in their Go trial response after they encounter a Stop error. This phenomenon, termed "post-error slowing", is a useful index of how well participants are monitoring their own performance. Both inhibitory control and performance monitoring are critical to cognitive control. Our participants performed the SST during fMRI, both at baseline and at 6 months.

\section{Imaging protocol}

Participants underwent fMRI during a resting state and while performing the stop signal task (as noted above). Functional blood oxygen level dependent (BOLD) signals were acquired using a $3 \mathrm{~T}$ scanner. Details of the imaging protocol and statistical modeling of brain images during stop signal task and at rest were as described previously [33]. Briefly, functional regions of interest were defined based on activated clusters from whole brain analysis. One of the main activated clusters was the medial prefrontal cortex (MPFC) which, as demonstrated in earlier work, plays a critical role in cognitive control $[34,35]$.

\section{Statistical analysis}

We estimated the sample size required to observe a group-by-time interaction effect on the basis of a previous pharmacological fMRI study [36]. The MPFC showed an effect size of 0.414 for the identical contrast in the stop signal task, according to Cohen's f-hat= square root of $\left[(\mathrm{Df} / \mathrm{N})^{*}(\mathrm{~F}-1)\right]$ [37]. The standard deviations for the interaction and within-cell error were 0.83 and 1.12, respectively. Using these results, and for a Type I error rate $=0.05$ as well as assuming a balanced design, a sample size of 16 in each cell will have a power of $85 \%$ to detect group by time interaction. In a region of interest analysis, we derived the effect size (extent) of MPFC activation of stop $>$ go trials [38]. Analyses were done based on the signals acquired; thus, "blinding" of results according to treatment group was not relevant. A significant effect of ADT would manifest as differences in regional brain activations for the contrast "(follow-up minus baseline in controls) $>$ (follow-up minus baseline among patients receiving ADT)". Signals obtained by fMRI from a task during rest can provide valuable information about the integrity of brain function, reflecting the intrinsic functional organization of the brain $[39,40]$.
We analyzed the resting state BOLD signals with the MPFC as the region of interest.

For further details on N-back working memory task, stop signal task, imaging protocol and imaging data analysis during stop signal task and resting state please see Additional file 1.

\section{Results}

From $01 / 2009$ to $1 / 2011,18$ patients with nonmetastatic prostate cancer receiving ADT and 18 control patients not receiving ADT were enrolled. One man died of a cause unrelated to prostate cancer and five withdrew their participation, resulting in 15 study participants undergoing ADT and 15 control participants (Table 1). These 30 study participants completed all scheduled assessments.

\section{Quality of life scores}

No statistically significant differences between ADT patients and control patients were found in QOL scores at baseline, or with regard to change over 6 months using the FACT-P ${ }^{\odot}$ questionnaire (Table 1). Of note, FACT-P ${ }^{\odot}$ does not specifically assess cognitive function.

Table 1 Patient characteristics and Quality of life rating

\begin{tabular}{|c|c|c|}
\hline Group & ADT $n=15$ & Control $n=15$ \\
\hline Age & $69.0 \pm 5.3 y$ & $66.1 \pm 6.2$ y \\
\hline \multirow[t]{5}{*}{ Education } & $9^{\text {th }}$ grade: 1 & $9^{\text {th }}$ grade: 1 \\
\hline & High School/GED: 5 & High School/GED: 5 \\
\hline & College 1-3 years: 3 & College 1-3 years: 3 \\
\hline & College graduate: 2 & College graduate: 3 \\
\hline & Post-graduate: 4 & Post-graduate: 3 \\
\hline MMSE & $29.0 \pm 1.3$ & $29.5 \pm 0.6$ \\
\hline \multirow[t]{3}{*}{ Cancer staging } & Stage II: 13 & Stage I: 1 \\
\hline & Stage III: 2 & Stage II: 12 \\
\hline & & Stage III: 2 \\
\hline \multirow[t]{3}{*}{ Local therapy } & Radiation 100\% & Radiation 26.7\% \\
\hline & & Surgery $66.7 \%$ \\
\hline & & Surgery + Radiation $6.7 \%$ \\
\hline QOL at baseline & $115 \pm 22$ & $132 \pm 18$ \\
\hline QOL of life at 6 months & $110 \pm 23$ & $128 \pm 21$ \\
\hline $\begin{array}{l}\text { Testosterone at } \\
6 \text { months }\end{array}$ & $0.14 \pm 0.10 \mathrm{ng} / \mathrm{ml}$ & $2.84 \pm 0.94 \mathrm{ng} / \mathrm{ml}$ \\
\hline
\end{tabular}

Note: ADT: Androgen Deprivation Therapy; GED: General Education Development Test; MMSE: Mini Mental State Examination; QOL: FACT-P quality of life score; there is no difference between groups in age $(t=1.172, p=0.588$, two-sided two-sample $t$ test); there is no difference between groups in MMSE score $(t=1.417, p=0.167$, two-sided two-sample $t$ test); staging follows the current guidelines of the 2010 American Joint Committee on Cancer (AJCC) that include Gleason score in the staging; there is also no difference across the two time points in the change of QOL between groups $(F=0.056, p=0.814$ interaction, repeated measures analysis of variance). 


\section{Cognitive performance in $\mathrm{N}$-back task and stop signal task}

As measured by the N-back task or by the stop-signaltask, no statistically significant differences in cognitive performance were observed at 6 months compared to baseline in either group (Tables 2 and 3 ).

Table 2 shows the results of N-back task performance scores. Overall, correct response rate decreased with increasing memory load for both control and ADT participants. Three-way repeated measures ANOVA with time (baseline vs. follow-up) and load (0-, 1-, and 2- back) as within-subject variables, and group (control vs. ADT) as the between-subject variable, showed that the interaction was not significant for hit rate $(\mathrm{p}=0.989)$ or reaction time (RT) $(\mathrm{p}=0.948)$ within the correct trials. Three-way repeated measures ANOVA with time (baseline vs. follow-up) and load (0- vs. 1- back, or 0- vs. 2-back) as within-subject variables, and group (control vs. ADT) as the between-subject variable, also showed that none of the interactions were significant $(\mathrm{p}=0.859$ and $\mathrm{p}=0.934$, respectively, for 0 - vs. 1 - back and 0 - vs. 2- back re: hit rate; $\mathrm{p}=0.814$ and $\mathrm{p}=0.732$, respectively, for 0 - vs. 1 back and 0- vs. 2- back re: RT of correct trials). We conducted two-way repeated measures ANOVA separately for 0 -, 1-, and 2- back data, which again yielded nonsignificant interactions (Table 2). These results indicated indistinguishable $\mathrm{N}$-back task performance between the two groups across the two time points.

Table 3 shows the performance outcomes of the stop signal task for the ADT and control group, at baseline and at 6 months. We recorded the Go success rate, Stop success rate, reaction time (RT) of Go success trials, the stop signal reaction time (SSRT), as well as the effect size of post-error slowing (i.e., how much participants slowed down in a Go trial following a Stop error), as an index of performance monitoring. The results showed that there were no differences in performance at 6 months compared to baseline in either group.

\section{Brain activations during cognitive control using the stop-signal-task}

Brain activations while performing the stop-signal-task were similar between baseline and follow-up for the control group. In contrast, regional brain activations were
Table 3 Behavioral performance in the stop signal task

\begin{tabular}{llllll}
\hline \multicolumn{5}{c}{ SSRT (ms) } & $\begin{array}{l}\text { Median go } \\
\text { RT }(\mathbf{m s})\end{array}$ \\
\hline ADT_B & $267 \pm 71$ & $697 \pm 127$ & $90.9 \pm 5.5$ & $56.5 \pm 6.0$ & $0.83 \pm 1.72$ \\
ADT_F & $278 \pm 37$ & $678 \pm 123$ & $89.6 \pm 5.2$ & $53.1 \pm 3.7$ & $1.21 \pm 1.12$ \\
Control_B & $260 \pm 57$ & $676 \pm 122$ & $91.6 \pm 5.9$ & $56.5 \pm 4.5$ & $1.73 \pm 1.56$ \\
Control_F & $268 \pm 56$ & $705 \pm 115$ & $91.2 \pm 5.4$ & $56.9 \pm 5.8$ & $1.49 \pm 1.35$ \\
P value* & 0.480 & 0.243 & 0.567 & 0.051 & 0.310 \\
\hline
\end{tabular}

Note: B: baseline; F: follow-up; SSRT: stop-signal reaction time; go RT: reaction time of correct go trials; \%go and \%stop: percentage of successful go and stop trials; PES: post-error slowing; all values are mean \pm standard deviation; *P value of the group by time interaction in repeated measures analysis of variance

significantly diminished in the ADT group at follow-up, as compared to baseline (Figure 1). These differences were most prominent in the medial prefrontal cortex (MPFC), right insula, and right middle/inferior frontal cortices. As previous work has demonstrated, the prefrontal cortex plays a critical role in cognitive control $[34,35]$. In a region of interest analysis, we focused on the MPFC and extracted the effect size of activation for individual participants. A repeated measures analysis of variance, with the ADT vs. control as the betweensubject factor and follow-up vs. baseline as the withinsubject factor, showed that ADT significantly decreased medial prefrontal cortical activation during cognitive control $\left(\mathrm{F}_{1,28}=7.903, \mathrm{p}=0.009\right.$, group by time interaction, Figure 2).

\section{Functional connectivity of medial prefrontal cortex during a resting state}

Despite performance scores on N-back task and stopsignal-task that were similar to the control patients, ADT patients showed decreased MPFC brain activations on fMRI at 6 months while performing the stop signal task. We were interested in whether changes on fMRI could also be observed when patients were not performing any cognitive tasks and studied the correlation of low frequency BOLD signals between the MPFC and other brain regions during a resting state. This resting state imaging reflects the functional connectivity between different brain regions and measures how well individual brain regions activate in a

Table 2 Performance in the N-back working memory task

\begin{tabular}{lllllll}
\hline & 0-back (correct \%) & 0-back (RT, ms) & 1-back (correct \%) & 1-back (RT, ms) & 2-back (correct \%) & 2-back (RT, ms) \\
\hline ADT_B & $98 \pm 3$ & $545 \pm 114$ & $85 \pm 13$ & $668 \pm 147$ & $63 \pm 15$ & $746 \pm 159$ \\
ADT_F & $97 \pm 5$ & $571 \pm 91$ & $86 \pm 11$ & $704 \pm 165$ & $64 \pm 17$ & $787 \pm 173$ \\
Control_B & $99 \pm 2$ & $491 \pm 88$ & $85 \pm 14$ & $585 \pm 157$ & $75 \pm 17$ & $677 \pm 152$ \\
Control_F & $99 \pm 1$ & $540 \pm 87$ & $89 \pm 3$ & $626 \pm 139$ & $78 \pm 13$ & $714 \pm 126$ \\
P value* & 0.402 & 0.389 & 0.577 & 0.932 & 0.493 & 0.920 \\
\hline
\end{tabular}

Note: $\mathrm{B}$ : baseline; $\mathrm{F}$ : follow-up; $\mathrm{RT}=$ reaction time of correct trials; ${ }^{\mathrm{P}} \mathrm{v}$ value of the group by time interaction in repeated measures analysis of variance. 


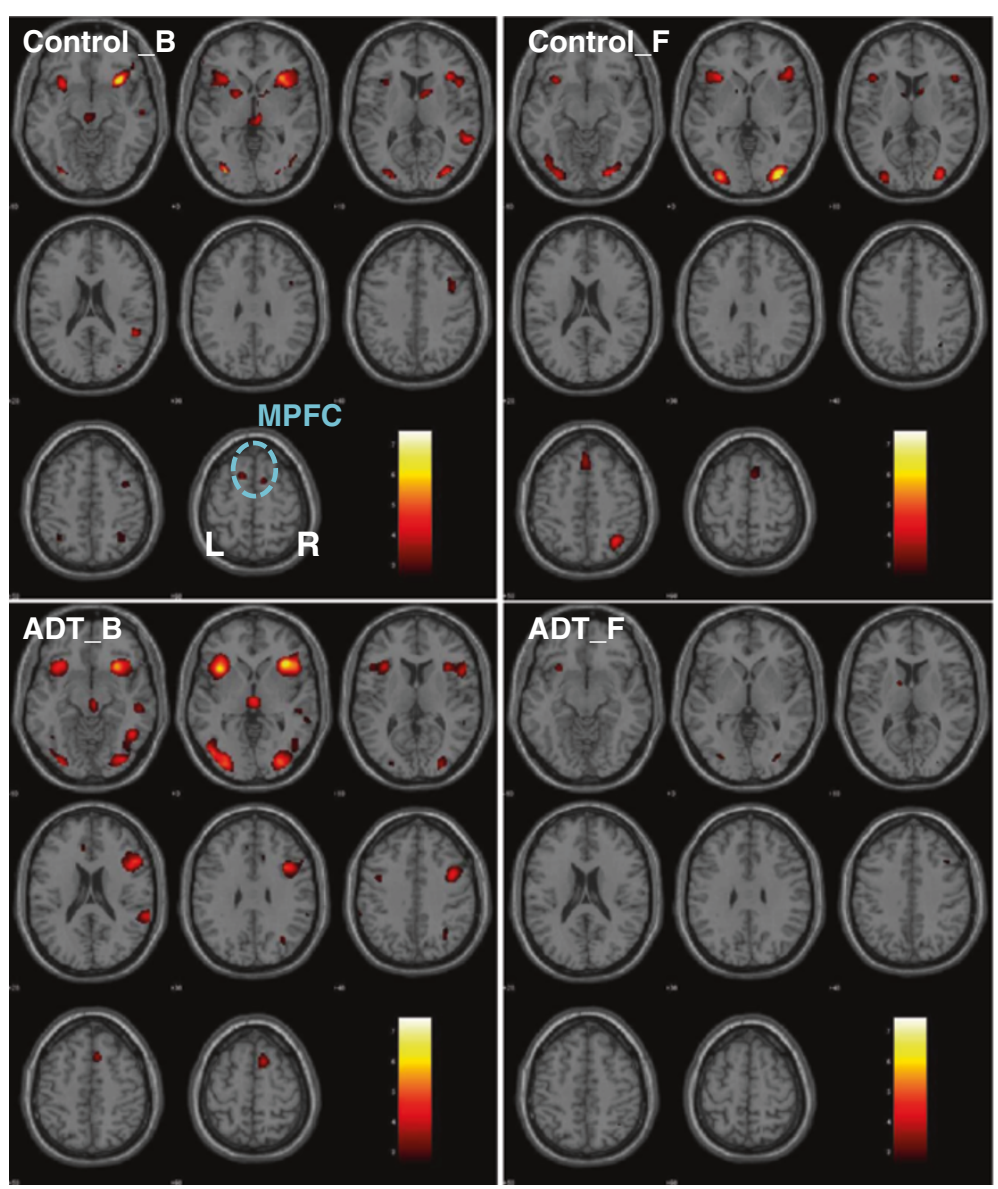

Figure 1 Regional brain activations during cognitive control while performing the stop signal task. ADT= patients who received 6 months of androgen deprivation therapy; Controls = patients who did not receive any hormonal therapy; $B=$ baseline; $F=$ at $6-$ month follow-up. The significance of activation, as reflected by a map of T values (color bar), is shown here on a structural brain image in axial sections, from $z=-10$ to $z=+60$, with adjacent sections $10 \mathrm{~mm}$ apart. Neurological orientation: Right $(\mathrm{R})=$ right. Note that ADT patients showed diminished activations in a number of brain regions at 6 month follow-up, including the medial prefrontal cortex (MPFC), an area critical for cognitive control.

concerted manner as an important index of the integrity of brain functions.

The results of an analysis of variance showed that, compared with the control group, ADT patients had at 6 months a decrease in MPFC connectivity $(\mathrm{p}<0.001$, uncorrected; Figure 3) with the dorsolateral prefrontal cortex (DLFPC), right insula, right superior temporal gyrus, and the rostral anterior cingulate cortex, structures that are broadly implicated in cognitive control.
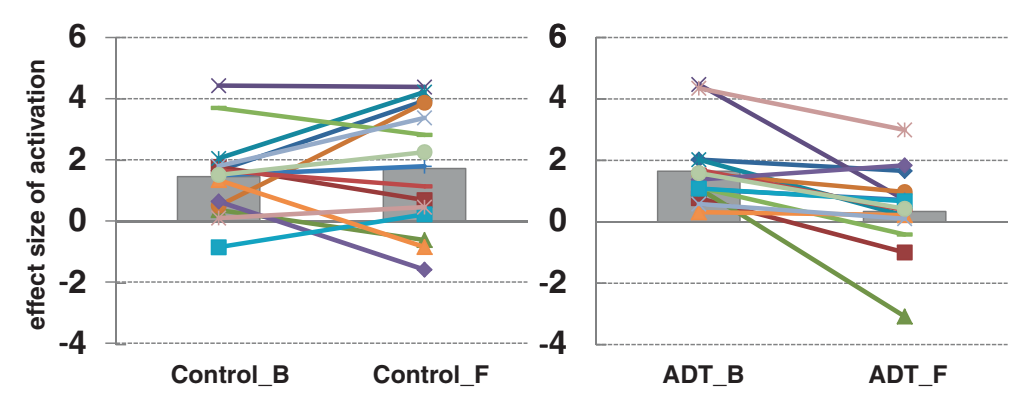

Figure 2 The effect size of medial prefrontal cortical (MPFC) activations. Control patients are shown on the left panel; ADT patients are shown on the right panel; $\mathrm{B}$ = baseline; $\mathrm{F}=$ at 6-month follow-up. In each panel, each symbol and line represents the data of an individual patient. The gray bars indicate the mean value of the effect sizes of MPFC activations. Individuals varied in the change of MPFC activations from baseline to follow-up, but, on average, the ADT group showed significant decrease in MPFC activations after 6 months of ADT, as compared to the control group. 

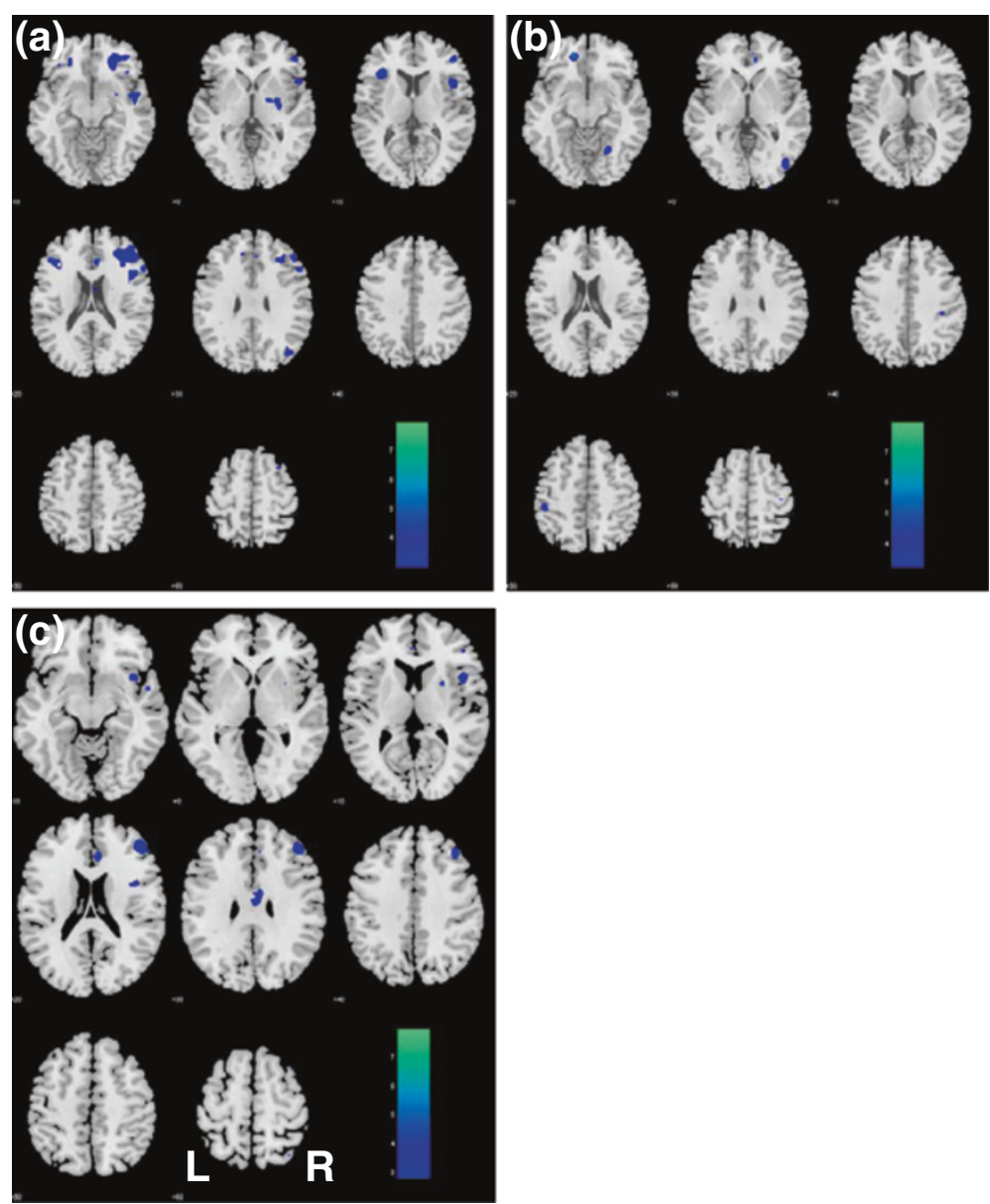

Figure 3 Changes in resting state functional connectivity with the medial prefrontal cortex as result of ADT. After 6 months of ADT, patients showed decreased connectivity with the dorsolateral prefrontal cortex (DLPFC), rostral anterior cingulate cortex, and the insula, all on the right side, as compared to control patients. (a) ADT_B > ADT_F; (b) Control_B > Control_F; (c) (Control_F>Control_B) - (ADT_F > ADT_B). B: baseline; F: follow-up. The significance of difference, as reflected by a map of T values (color bar), is shown here on a structural brain image in axial sections, from $z=-10$ to $z=+60$, with adjacent sections $10 \mathrm{~mm}$ apart. Neurological orientation: Right $(\mathrm{R})=$ right.

\section{Discussion}

Studies of traditional neurocognitive testing without brain imaging have shown equivocal impact of $\mathrm{ADT}$ on cognitive function. In the current study, we found suppressive effects on brain activations and disruption in functional connecticity on fMRI in prostate cancer patients after 6 months of ADT. Interestingly, performance in neurocognitive testing and their QOL scores remained similar compared to their baseline, and control patients without ADT did not show any fMRI changes. Our patients were matched by age and level of education, and all assessments were performed either before or at least 3 months after any surgery or radiation treatment to minimize any impact of treatment-related symptoms.

The stability of cognitive performance scores after six months of ADT is consistent with a recent report [41] that used neurocognitive tesing alone in a larger sample of patients receiving twelve months of ADT. In contrast, the changes we found for brain activations on fMRI while performing the stop-signal-task after 6 months of ADT were substantial.

Previous work has demonstrated that the MPFC and a network of brain regions interacting with the MPFC play a critical role during cognitive control $[34,35]$. The findings of altered MPFC activation following ADT are consistent with previous literature on the modulatory effects of androgen on cerebral cortical activities [42-46]. For example, a higher level of free testosterone was associated with greater cerebral blood flow in the hippocampus and prefrontal cortices in elderly men [45], testosterone replacement therapy increased cerebral blood perfusion in the midbrain and prefrontal cortex in hypogonadal men [42], and administration of testosterone increased ventral striatal responses to reward in healthy women [44]. A preliminary report of decreased 
parieto-occipital brain activation during visuospatial processing in five men undergoing ADT [47] is consistent with our results in suggesting a suppressive effect of ADT on brain activity.

As mentioned previously, neuroimaging studies have reported differences in regional brain activations in neurological or psychiatric patients even when they performed at a level equal to their demographically-matched control participants. These performance-independent changes in brain activities cannot be accounted for by effort or motivation, and potentially represent the neural correlates specific to the cerebral pathologies. Similarly, the findings of decreased cortical activations in prostate cancer patients undergoing ADT, despite similar performance scores on neurocognitive testing, indicate that changes in brain activations were not a result of these patients being less engaged in the task, as compared with their counterparts. Furthermore, neurocognitive performance is potentially subject to practice effects whereby participants' performance improves upon repeated testing. Performance-independent differences in brain activations may thus be more objective and reveal subtle changes in brain function that cannot be measured by neurocognitive testing alone.

Limitations of this study include a small sample size and a limited duration of ADT exposure, yet we were able to demonstrate an impact of therapy. Although our study design supports the validity of our results, the results should be considered preliminary with the need for replication in future work. Our study is distinctive in evaluating the effects of ADT using functional imaging; it also is novel in demonstrating a disruption of functional connectivity at rest in patients treated with ADT. In addition, although our study was not a randomized trial, the potential impact of ADT on brain activation should be detectable using either randomized or observational approaches. Finally, our study cannot answer questions such as whether the differences observed using fMRI would worsen over time with or without continued ADT, or whether even these current effects would eventually lead to clinically apparent signs or symptoms. Future studies with longer follow-up are warranted to answer these important questions.

\section{Conclusion}

ADT limited to 6 months did not affect scores on selected tests of cognitive function. However, using sensitive techniques involving fMRI, ADT for 6 months suppressed brain activations during cognitive control and disrupted brain functional connectivity in prostate cancer patients. The long-term clinical implications of these changes are not known and warrant future study.

\section{Additional file}

Additional file 1: Supplemental material.

\section{Competing interests}

The authors declare that they have no competing interests.

\section{Authors' contributions}

$\mathrm{HHC}$ conceived of the study, participated in its design and coordination and drafted the manuscript. EU participated in the design and enrollment of patients and helped to draft the manuscript. SZ, SH and SRB participated in the coordination of the study and performed the statistical analysis of neurocognitive testing. $\mathrm{XL}$ performed the analysis of imaging data. MR and JC participated in the design of the study and the writing of the manuscript. CRL participated in the design of the study, data analysis and writing of the manuscript. All authors read and approved the final manuscript.

\section{Acknowledgements}

This study was supported by NIH grant R03CA138121 to H.H.C., a Yale Cancer Center/Connecticut Challenge Survivorship pilot award and VA Urology Research Sectional Funding. We thank Monica Delvy and George Hayner for their assistance in coordinating this study. The funding agencies had no further role in study design; in the collection, analysis and interpretation of data; in the writing of the report; or in the decision to submit the paper for publication. All authors have no financial interests to disclose.

\section{Author details}

'Department of Internal Medicine \& Yale Comprehensive Cancer Center, Yale University School of Medicine, New Haven, CT 06519, USA. ${ }^{2}$ Medical Service, VA Connecticut Health Care System, West Haven, CT 06516, USA. ${ }^{3}$ Tower Research, Los Angeles, CA 90048, USA. ${ }^{4}$ Department of Psychiatry, Yale University School of Medicine, New Haven, CT 06519, USA. ${ }^{5}$ Department of Biostatistics, Brown University School of Medicine, Providence, RI 02912, USA. ${ }^{6}$ Clinical Epidemiology Research Center, West Haven, CT 06516, USA. ${ }^{7}$ Department of Neurobiology, Yale University School of Medicine, New Haven, CT 06520, USA. ${ }^{8}$ Interdepartment Neuroscience Program, Yale University, New Haven, CT 06520, USA. ${ }^{9}$ Cancer Center, VA Connecticut Health Care System, 950 Campbell Avenue, West Haven, CT 06516, USA.

Received: 2 March 2012 Accepted: 21 August 2012

Published: 27 August 2012

\section{References}

1. Meng MV, Grossfeld GD, Sadetsky N, Mehta SS, Lubeck DP, Carroll PR: Contemporary patterns of androgen deprivation therapy use for newly diagnosed prostate cancer. Urology 2002, 60(Suppl 3A):7-12.

2. Shahinian VB, Kuo Y-F, Freeman JL, Orihuela E, Goodwin JS: Increasing use of gonadotropin-releasing hormone agonists for localized prostate cancer. Cancer 2005, 103:1615-1624.

3. Alibhai SM, Gogov S, Allibhai Z: Long-term side effects of androgen deprivation therapy in men with non-metastatic prostate cancer: a systematic literature review. Crit Rev Oncol Hematol 2006, 60:201-215.

4. Moffat SD, Zonderman AB, Metter EJ, Kawas C, Blackman MR, Harman SM, Resnick SM: Free testosterone and risk for Alzheimer disease in older men. Neurology 2004, 62:188-193.

5. Kenny AM, Fabregas G, Song C, Biskup B, Bellantonio S: Effects of testosterone on behavior, depression, and cognitive function in older men with mild cognitive loss. J Gerontol A Biol Sci Med Sci 2004, 59:75-78.

6. Cherrier MM, Matsumoto AM, Amory JK, Johnson M, Craft S, Peskind ER, Raskind MA: Characterization of verbal and spatial memory changes from moderate to supraphysiological increases in serum testosterone in healthy older men. Psychoneuroendocrinology 2007, 32:72-79.

7. Cherrier MM, Matsumoto AM, Amory JK, Asthana S, Bremner W, Peskind ER, Raskind MA, Craft S: Testosterone improves spatial memory in men with Alzheimer disease and mild cognitive impairment. Neurology 2005, 64:2063-2068.

8. Lu PH, Masterman DA, Mulnard R, Cotman C, Miller B, Yaffe K, Reback E, Porter V, Swerdloff R, Cummings JL: Effects of testosterone on cognition 
and mood in male patients with mild Alzheimer disease and healthy elderly men. Arch Neurol 2006, 63:177-185.

9. Nelson CJ, Lee JS, Gamboa MC, Roth AJ: Cognitive effects of hormone therapy in men with prostate cancer: a review. Cancer 2008, 113(5):1097-1106.

10. Joly F, Alibhai SM, Galica J, Park A, Yi QL, Wagner L, Tannock IF: Impact of androgen deprivation therapy on physical and cognitive function, as well as quality of life of patients with nonmetastatic prostate cancer. J Urol 2006, 176:2443-2447.

11. Almeida OP, Waterreus A, Spry N, Flicker L, Martins RN: One year follow-up study of the association between chemical castration, sex hormones, beta-amyloid, memory and depression in men. Psychoneuroendocrinology 2004, 29:1071-1081.

12. Green HJ, Pakenham Kl, Headley BC, Yaxley J, Nicol DL, Mactaggart PN, Swanson C, Watson RB, Gardiner RA: Altered cognitive function in men treated for prostate cancer with luteinizing hormone-releasing hormone analogues and cyproterone acetate: a randomized controlled trial. BJU Int 2002, 90:427-432.

13. Jenkins VA, Bloomfield DJ, Shilling VM, Edginton TL: Does neoadjuvant hormone therapy for early prostate cancer affect cognition? Results from a pilot study. BJU Int 2005, 96:48-53.

14. Salminen $E$, Portin R, Korpela J, et al: Androgen deprivation and cognition in prostate cancer. Br J Cancer 2004, 89:971-976.

15. Miller SP, McQuillen PS: Neurology of congenital heart disease: insight from brain imaging. Arch Dis Child Fetal Neonatal Ed 2007, 92:F435-F437.

16. Korzeniewski SJ, Birbeck G, DeLano MC, Potchen MJ, Paneth N: A systematic review of neuroimaging for cerebral palsy. J Child Neurol 2008, 23:216-227.

17. Tan HY, Sust S, Buckholtz JW, Mattay VS, Meyer-Lindenberg A, Egan MF, Weinberger DR, Callicott JH: Dysfunctional prefrontal regional specialization and compensation in schizophrenia. Am J Psychiatry 2006, 163:1969-1977.

18. Driesen NR, Leung HC, Calhoun VD, Constable RT, Gueorguieva R, Hoffman R, Skudlarski P, Goldman-Rakic PS, Krystal JH: Impairment of Working Memory Maintenance and Response in Schizophrenia: Functional Magnetic Resonance Imaging Evidence. Biol Psychiatry 2008, 64:1026-1034

19. Koch K, Wagner G, Nenadic I, Schachtzabel C, Schultz C, Roebel M, Reichenbach JR, Sauer H, Schlosser RG: Fronto-striatal hypoactivation during correct information retrieval in patients with schizophrenia: an fMRI study. Neuroscience 2008, 153:54-62.

20. Castellon SA, Silverman DH, Ganz PA: Breast cancer treatment and cognitive functioning: current status and future challenges in assessment. Breast Cancer Res Treat 2005, 92:199-206.

21. Silverman DH, Dy CJ, Castellon SA, Lai J, Pio BS, Abraham L, Waddell K, Petersen L, Phelps ME, Ganz PA: Altered frontocortical, cerebellar, and basal ganglia activity in adjuvant-treated breast cancer survivors 510 years after chemotherapy. Breast Cancer Res Treat 2007, 103(3):303-311.

22. Ferguson RJ, McDonald BC, Saykin AJ, Ahles TA: Brain structure and function differences in monozygotic twins: possible effects of breast cancer chemotherapy. J Clin Oncol 2007, 25(25):3866-3870.

23. Eberling JL, Wu C, Tong-Turnbeaugh R, Jagust WJ: Estrogen- and tamoxifen-associated effects on brain structure and function. Neurolmage 2004, 21:364-371.

24. Deprez S, Amant F, Smeets A, Peeters R, Leemans A, Van Hecke W, Verhoeven JS, Christiaens MR, Vandenberghe J, Vandenbulcke M, Sunaert S: Longitudinal assessment of chemotherapy-induced structural changes in cerebral white matter and its correlation with impaired cognitive functioning. J Clin Oncol 2012, 30(3):274-281. Epub 2011 Dec 19.

25. Kesler SR, Kent JS, O'Hara R: Prefrontal cortex and executive function impairments in primary breast cancer. Arch Neurol 2011, 68(11):1447-1453.

26. Folstein MF, Folstein SE, McHugh PR: "Mini-mental state". A practical method for grading the cognitive state of patients for the clinician. J Psychiatr Res 1975, 12:189-198.

27. First MB, Spitzer RL, Williams JBW, Gibbon M: Structured Clinical Interview for DSM-IV (SCID). Washington DC: American Psychiatric Association; 1995.

28. Esper P, Mo F, Chodak G, Sinner M, Cella D, Pienta KJ: Measuring quality of life in men with prostate cancer using the functional assessment of cancer therapy-prostate instrument. Urology 1997, 50:920-928.

29. Baddeley A: Working memory: looking back and looking forward. Nature Reviews. Neuroscience 2003, 4:829-839.
30. Kirchner WK: Age differences in short-term retention of rapidly changing information. J Exp Psychol 1958, 55:352-358.

31. Logan GD, Cowan WB, Davis KA: On the ability to inhibit simple and choice reaction time responses: a model and a method. J Exp Psychol Hum Percept Perform 1984, 10:276-291.

32. Li C-SR, Chao HH-A, Lee TW: The neural correlates of speeded compared to delayed responses in a stop signal task: an indirect analogue of risk taking and association with an anxiety trait. Cerebral Cortex 2009, 19:839-848.

33. Chao HH, Luo X, Chang JL, Li CS: Activation of the pre-supplementary motor area but not inferior prefrontal cortex in association with short stop signal reaction time-an intra-subject analysis. BMC Neurosci 2009, 10:75

34. Ridderinkhof KR, van den Wildenberg WP, Segalowitz SJ, Carter CS: Neurocognitive mechanisms of cognitive control: the role of prefrontal cortex in action selection, response inhibition, performance monitoring and reward-based learning. Brain Cogn 2004, 56:129-140.

35. Rushworth MF: Intention, choice, and the medial frontal cortex. Ann N Y Acad Sci 2008, 1124:181-207.

36. Li CS, Morgan PT, Matuskey D, Abdelghany O, Luo X, Chang JL, Rounsaville BJ, Ding YS, Malison RT: Biological markers of the effects of intravenous methylphenidate on improving inhibitory control in cocaine-dependent patients. Proc Natl Acad Sci U S A 2010, 107:14455-14459.

37. Cohen J: Statistical Power Analysis for the Behavioral Sciences (second edition). Hillsdale, New Jersey: Lawrence Erlbaum Associates; 1988

38. Brett M, Anton J-L, Valabregue R, Poline J-P: Region of interest analysis using an SPM toolbox. Abstract presented at the $8^{\text {th }}$ International Conference on Functional Mapping of the Human Brain. June 2-6. Sendai, Japan: 2002. Available on CD-ROM in Neuro-Image, 16(2), abstract 497.

39. Fair DA, Schlaggar BL, Cohen AL, Miezin FM, Dosenbach NU, Wenger KK, Fox MD, Snyder AZ, Raichle ME, Petersen SE: A method for using blocked and event-related fMRI data to study "resting state" functional connectivity. Neurolmage 2007, 35:396-405.

40. Fox MD, Raichle ME: Spontaneous fluctuations in brain activity observed with functional magnetic resonance imaging. Nat Rev Neurosci 2007 8:700-711

41. Alibhai SM, Breunis H, Timilshina N, Marzouk S, Stewart D, Tannock I, Naglie G, Tomlinson G, Fleshner N, Krahn M, Warde P, Canning SD: Impact of androgen-deprivation therapy on cognitive function in men with nonmetastatic prostate cancer. J Clin Oncol 2010, 28(34):5030-5037.

42. Azad N, Pitale S, Barnes WE, Friedman N: Testosterone treatment enhances regional brain perfusion in hypogonadal men. $J$ Clin Endocrinol Metab 2003, 88:3064-3068

43. Forbes EE, Ryan ND, Phillips ML, Manuck SB, Worthman CM, Moyles DL, Tarr JA, Sciarrillo SR, Dahl RE: Healthy adolescents' neural response to reward: associations with puberty, positive affect, and depressive symptoms. J Am Acad Child Adolesc Psychiatry 2010, 49:162-72.e1-5.

44. Hermans EJ, Bos PA, Ossewaarde L, Ramsey NF, Fernández G, van Honk J: Effects of exogenous testosterone on the ventral striatal BOLD response during reward anticipation in healthy women. Neurolmage 2010, 52:277-283.

45. Moffat SD, Resnick SM: Long-term measures of free testosterone predict regional cerebral blood flow patterns in elderly men. Neurobiol Aging 2007, 28:914-920.

46. van Wingen GA, Zylicz SA, Pieters S, Mattern C, Verkes RJ, Buitelaar JK, Fernández G: Testosterone increases amygdala reactivity in middle-aged women to a young adulthood level. Neuropsychopharmacology 2009, 34:539-547.

47. Cherrier MM, Borghesani PR, Shelton AL, Higano CS: Changes in neuronal activation patterns in response to androgen deprivation therapy: a pilot study. BMC Cancer 2010, 10:1

doi:10.1186/1471-2407-12-371

Cite this article as: Chao et al:: Effects of androgen deprivation on brain function in prostate cancer patients - a prospective observational cohort analysis. BMC Cancer 2012 12:371. 